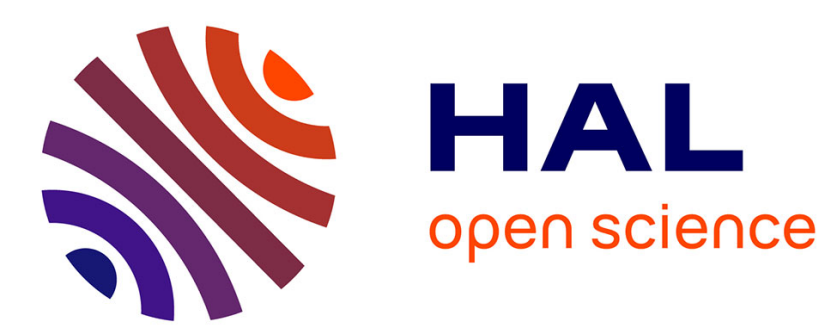

\title{
Les Agromyzidae de l'île de Saint-Christopher (Saint-Kitts) aux Antilles (Diptera)
}

\author{
M. Martinez
}

\section{To cite this version:}

M. Martinez. Les Agromyzidae de l'île de Saint-Christopher (Saint-Kitts) aux Antilles (Diptera).

Bulletin de la Société Entomologique de France, 2003, 108 (1), pp.89-95. hal-02673588

\section{HAL Id: hal-02673588 \\ https://hal.inrae.fr/hal-02673588}

Submitted on 31 May 2020

HAL is a multi-disciplinary open access archive for the deposit and dissemination of scientific research documents, whether they are published or not. The documents may come from teaching and research institutions in France or abroad, or from public or private research centers.
L'archive ouverte pluridisciplinaire HAL, est destinée au dépôt et à la diffusion de documents scientifiques de niveau recherche, publiés ou non, émanant des établissements d'enseignement et de recherche français ou étrangers, des laboratoires publics ou privés. 


\title{
Les Agromyzidae de l'île de Saint-Christopher (Saint-Kitts) aux Antilles (Diptera)
}

\author{
par Jean ETIENNE* et Michel MARTINEZ** \\ * INRA - URPV, laboratoire d'Entomologie, Lutte Intégrée, Domaine Duclos, F - 97170 Petit-Bourg \\ (Guadeloupe)<jean.etienne2@wanadoo.fr> ** INRA, UFR d'Ecologie animale et Zoologie \\ agricole, 2 place Pierre Viala, F - 34060 Montpellier cedex 01 <martinez@ensam.inra.fr>
}

Résumé. - Toutes les espèces de Diptères Agromyzidae actuellement connues de Saint-Christopher (Saint-Kitts) sont répertoriées. Sur les 38 espèces recensées, 37 sont nouvelles pour cette île, et parmi celles-ci, 5 le sont probablement aussi pour la science mais ne sont pas décrites ici. Quatre espèces d'importance agronomique sont signalées, il s'agit de Liriomyza brassicae, L. sativae, L. trifolii et de Nemorimyza maculosa. Des informations générales sont données sur la distribution géographique et les plantes-hôtes de chacune des espèces.

Summary. - The Agromyzidae of the Island of Saint-Christopher (Saint-Kitts) in the Antilles (Diptera). All species of Agromyzidae Diptera now known on Saint-Christopher (Saint-Kitts) are listed. Among the 38 species mentioned, 37 are new for this Island and 5 are probably new to science but they are not described here. Four species of agronomic importance Liriomyza brassicae, L. sativae, L. trifolii and Nemorimyza maculosa are indicated. For each species, general data are given on biogeography and host plants.

Mots clés. - Diptera, Agromyzidae, inventaire, ravageurs, Antilles, île de Saint-Christopher, Saint-Kitts.

Dans le cadre des études de faunistiques et de biosystématiques que nous menons sur les Agromyzidae de la région caraỉbe, l'un d'entre nous (J. E.) a effectué, à Saint-Christopher, diverses collectes de ces Diptères lors de deux brefs séjours (en 1993/1994 et 1996). Cette petite île antillaise de $169 \mathrm{~km}^{2}$, appelée plus communément Saint-Kitts se trouve au nordouest d'Antigua, entre Nevis au sud et Saba au nord.

Aucune recherche n'ayant été réalisée auparavant à Saint-Kitts, les 240 spécimens collectés ont permis de recenser 38 espèces dont 37 nouvelles pour cette île. Cinq de ces espèces sont probablement nouvelles pour la science, mais ne sont pas décrites ici en l'absence de matériel suffisant et par manque d'indications biologiques.

Il est certain que le nombre d'espèces d'Agromyzidae présentes à Saint-Christopher doit être beaucoup plus important car peu de localités ont été prospectées (seulement 5) et la majorité de nos collectes proviennent d'une seule d'entre elles (Fairview Inn). Il faut considérer ces récoltes comme un premier sondage, néanmoins fort intéressant car, comme pour l'île de Saint-Martin (ETIENNE \& MARTINEZ, 1996), la plupart des espèces sont connues de Guadeloupe et notamment celles qui y ont été décrites récemment in SPENCER et al., 1992.

Les principaux ravageurs d'importance agronomique comme les espèces polyphages Liriomyza sativae Blanchard, L. trifolii (Burgess) ou encore L. brassicae (Riley), cette dernière nuisible essentiellement aux Crucifères, ont été recensés à Saint-Kitts. L'espèce Nemorimyza maculosa (Malloch) a également été trouvée, elle peut, dans certaines conditions, être très nuisible aux jeunes plantations de laitue. Enfin signalons la présence sur l'île de Japanagromyza etiennei décrit récemment (MARTINEZ, 1994) et de Liriomyza marginalis (Malloch) qui peuvent être considérés comme des ravageurs potentiels respectivement de Fabaceae (genres Phaseolus et Vigna) et de Poaceae (genre Zea). A notre connaissance ces espèces d'intérêt agronomique n'ont fait l'objet d'aucun travail à Saint-Kitts. On trouvera cependant dans diverses publications concernant la Guadeloupe ou la Caraibe (MARTINEZ \& ETIENNE, 199.1, SPENCER et al., 1992, ETIENNE et al., 1993 et MARTINEZ et al., 1993) des données agronomiques les concernant. 
Notes : les genres et les espèces, au nombre de 38 , sont listés à la suite, par ordre alphabétique; rappelons que 37 d'entres elles sont nouvelles pour l'île de Saint-Christopher.

Parmi les spécimens capturés au fauchage, seuls les mâles ont été déterminés car en l'absence d'indications biologiques (plantes-hôtes), l'identification des femelles est souvent aléatoire voire impossible.

Les plantes-hôtes découvertes récemment (ETIENNE \& MARTINEZ, 2002). sont précédées d'un astérisque. Les larves mineuses de feuilles, cas le plus fréquent, sont signalées par un "M".

\section{1 - Agromyza sp.}

Genre Agromyza Fallén, 1810

Matériel examiné: Fairview Inn, 5.I.1994, 1 \% , au fauchage.

Dans la clé d'identification des Agromyza de Floride (SPENCER \& STEGMAIER, 1973: 18) cette espèce, au $3^{\mathrm{e}}$ article antennaire noir, est proche de l'Agromyza sp. (loc. cit., p. 22). Elle en diffère cependant par ses joues plus larges et le nombre $(3+1)$ et la taille respective de ses soies dorsocentrales. Du matériel complémentaire est nécessaire pour savoir s'il s'agit d'une espèce déjà décrite ou non.

\section{Genre Calycomyza Hendel, 1931}

\section{2 - Calycomyza addita Spencer}

Calycomyza addita Spencer, 1983:60.

Matériel examiné: Fairview Inn, 3.I.1994, 1 ơ $^{\star}$ au fauchage.

Distribution: Costa Rica, Guadeloupe.

Plantes-hôtes: *Tecoma stans (L.) [Bignoniaceae], M.

3 - Calycomyza bahamarum Spencer

Calycomyza bahamarum Spencer: 182 (in SPENCER \& STEGMAIER, 1973).

Matériel examiné: Lynshes Gauht, 31.XII.1993, 20, au fauchage.

Distribution: Bahamas, Cuba, Guadeloupe.

Plantes-hôtes: Lantana reticulata Pers. et Stachytarpheta jamaicensis (L.) [Verbenaceae], M.

4 - Calycomyza caguensis Spencer

Calycomyza caguensis Spencer, 1973b: 38 .

Matériel examiné: Fairview Inn, 1, 3 et 5.I.1994, 40, au fauchage.

Distribution: Costa Rica, Guadeloupe, Venezuela.

Plante-hôte: *Synedrella nodiflora (L.) [Asteraceae], M.

\section{5 - Calycomyza ipomaeae (Frost)}

Agromyza ipomaeae Frost, 1931:74.

Matériel examiné: Fairview Inn, 1.I.1994, 20 , au fauchage.

Distribution: Barbade, Brésil, Chili, Colombie, Cuba, Guadeloupe, Grand-Cayman, Jamaïque, Dominique, Nicaragua, Pérou, Porto Rico, République Dominicaine, Saint-Martin, Venezuela, Canada, USA (Floride).

Plantes-hôtes:Ipomoea spp. et *Stictocardia tiliaefolia (Desr.) [Convolvulaceae], M.

\section{6 - Calycomyza ipomoeaphaga Martinez}

Calycomyza ipomoeaphaga, Martinez: 289 (in Spencer et al., 1992).

Matériel examiné: Fairview Inn, 3.I.1994, 1 đ, au fauchage.

Distribution: Guadeloupe, Saint-Martin.

Plantes-hôtes: Ipomoea spp., $\mathbf{M}$.

\section{7 - Calycomyza sp. 1, groupe malvae - sidae}

Matériel examiné : Fairview Inn, 31.XII.1993, $1 \delta^{\star}$; Lynshes Gauht, même date, $1 \delta^{\text {đ }}$; au fauchage. Note : ce groupe nécessite une révision et dans l'état actuel nous hésitons à nommer ces spécimens. 


\section{8 - Calcomyza sp. 2}

Matériel examiné : Brimstone Hill, 31.XII.1993, 10 , au fauchage.

Note: cet exemplaire correspond à un spécimen unique capturé en Guadeloupe. Il s'agit d'une espèce manifestement nouvelle; par son édéage elle fait partie du groupe $C$. verbesinae (Spencer).

9 - Haplopeodes sp.

Genre Haplopeodes Steyskal, 1980

Matériel examiné : Brimstone Hill, 31.XII.1993, 50 et 2 ㅇ ; Basse-Terre, 4.VI.1996. Tous ces spécimens obtenus de la plante-hôte.

Distribution: Guadeloupe, Saint-Martin.

Plante-hôte : *Solanum racemosum Jacq. [Solanaceae], M.

Note: il est probable que cette espèce soit nouvelle et son étude est en cours.

\section{Genre Japanagromyza Sasakawa, 1958}

10 - Japanagromyza bennetti Spencer

Japanagromyza bennetti Spencer: 145 (in SPENCER \& STEGMAIER, 1973).

Matériel examiné : Fairview Inn, 4.I.1994, 1 ơ $^{\circ}$ au fauchage.

Distribution: Antigua, Barbade, Guadeloupe, Porto Rico.

Plante-hôte: *Rhynchosia phaseoloides (SW) [Fabaceae], M.

11 - Japanagromyza centrosematifolii Etienne

Japanagromyza centrosematifolii Etienne, 2002 (in ETIENNE \& MARTINEZ, 2002).

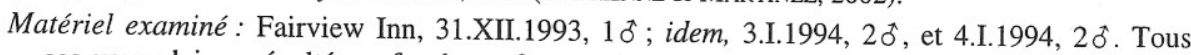
ces exemplaires, récoltés au fauchage, font partie de la série typique.

Distribution: Guadeloupe, Martinique, Saint-Barthélemy.

Plantes-hôtes: *Centrosema pubescens Benth. et C. virginianum (L.) [Fabaceae], M.

\section{2 - Japanagromyza desmodivora Spencer}

Japanagromyza desmodivora Spencer, 1996:4.

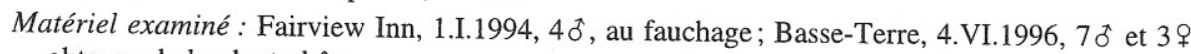
obtenus de la plante-hôte.

Distribution: Equateur, Guadeloupe, Pérou, Venezuela, USA (Floride).

Plante-hôte: Desmodium tortuosum (SW) [Fabaceae], M.

\section{3 - Japanagromyza etiennei Martinez}

Japanagromyza etiennei Martinez, 1994: 82.

Matériel examiné : Lynshes Gauht, 31.XII.1993, $20^{\star}$ et 5 \% Rappelons que des paratypes de cette espèce proviennent de Saint-Christopher.

Distribution: Guadeloupe, Jamaïque, Saint-Martin.

Plantes-hôtes: Phaseolus lunatus L. et *Vigna unguiculata (L.) [Fabaceae], M.

\section{4 - Japanagromyza inferna Spencer}

Japanagromyza inferna Spencer: 147 (in SPENCER \& STEGMAIER, 1973).

Matériel examiné : Basse-Terre, 4.VI.1996, 4 đ et 1 \% obtenus de la plante-hôte.

Distribution: Bahamas, Guadeloupe.

Plante-hôte: *Centrosema virginianum (L) [Fabaceae], la larve fait une galle foliaire.

\section{5 - Japanagromyza perpetua Spencer}

Japanagromyza perpetua Spencer: 28 (in SPENCER \& STEGMAIER, 1973).

Matériel examiné : Stone Fort River, Bloody Point, 31.XII.1993, 40̋ ; Fairview Inn, 31.XII.1993, $2 \delta^{\dagger}$; idem, 1.I.1994, 7ठే; idem, 5.I.1994, $10 \delta^{\star}$. Tous au fauchage.

Distribution: Bahamas, Costa Rica, Guadeloupe, Dominique, Porto Rico, République Dominicaine, Saint-Martin, USA (Floride).

Plante-hôte: *Desmodium tortuosum (SW) [Fabaceae], la larve se développe dans les gousses. 
Genre Liriomyza Mik, 1894

16 - Liriomyza blechi Spencer

Liriomyza blechi Spencer : 98 (in SPENCER \& STEGMAIER, 1973).

Matériel examiné : Stone Fort River, Bloody Point, 31.XII.1993, 1 đ̊ ; Fairview Inn, 3.XII.1994, 10 ; idem, 4.I.1994, 20 . Tous au fauchage.

Distribution: Guadeloupe, République Dominicaine, USA (Floride).

Plantes-hôtes: Blechum brownei Juss. (= B. pyramidatum Urb.) [Acanthaceae], M.

17 - Liriomyza brassicae (Riley)

Oscinis brassicae Riley, 1885:322.

Matériel examiné: Fairview Inn, 1.I.1994, 10ึ; idem, 3.I.1994, 30ิ; idem, 4.I.1994, 10ิ. Tous au fauchage. Basse-Terre, 4.VI.1996, $1 \delta^{\text {ț }}$ et $1 \%$, obtenus de Cleome viscosa L. [Capparidaceae].

Distribution: Argentine, Brésil, Guyana, Guadeloupe, Martinique, Venezuela; régions afrotropicale, australienne/pacifique, néarctique, orientale et paléarctique.

Plantes-hôtes: principalement des crucifères [Brassicaceae] avec les genres cultivés Brassica, Cheiranthus, Lepidium, Raphanus, Roripa, Barbarea, Cakile, Eruca, Hirschfeldia, Isatis, Matthiola, Moricandia; des Capparidaceae, Capparis, Cleome; des Tropaeolaceae, Tropaeolum. M.

18 - Liriomyza commelinae (Frost)

Agromyza commelinae Frost, 1931:72.

Matériel examiné : Fairview Inn, 31.XII.1993, 1 đo au fauchage.

Distribution: Argentine, Barbade, Brésil, Colombie, Costa Rica, Cuba, Guadeloupe, Jamaïque, Pérou, Saint-Martin, Saint-Vincent, Venezuela, USA (Floride).

Plantes-hôtes: Commelina spp. et Tradescantia fluminensis Vell. [Commelinaceae], $\mathbf{M}$.

19 - Liriomyza guadeloupensis Martinez

Liriomyza guadeloupensis Martinez: 278 (in SPENCER et al., 1992).

Matériel examiné : Fairview Inn, 1, 3, 4 et 5.I.1994, 14ðे, au fauchage. Fairview Inn, 5.I.1994, $3{ }^{\dagger}$ et 3 o obtenus de Tridax procumbens L. [Asteraceae].

Distribution: Guadeloupe, Saint-Martin.

Plantes-hôtes: *Ageratum conyzoides L., *Galinsoga sp. et *Tridax procumbens L. [Asteraceae], M.

20 - Liriomyza marginalis (Malloch)

Agromyza melampyga var. marginalis Malloch, 1913:283.

Matériel examiné : Stone Fort River, Bloody Point, 31.XII.1993, 1 ơ ; Fairview Inn, 1, 3, 4 et 5.I. 1994, $180^{\star}$. Tous au fauchage.

Distribution: Bahamas, Brésil, Chili, Colombie, Costa Rica, Cuba, Guadeloupe, Jamaïque, Dominique, Nicaragua, Pérou, Saint-Martin, Venezuela, USA (Floride).

Plantes-hôtes: diverses Poaceae appartenant aux genres Digitaria, Eleusine, Euchlaena, Panicum, Paspalum et Zea, M.

21 - Liriomyza sativae Blanchard

Liriomyza sativae Blanchard, 1938: 354.

Matériel examiné : Fairview Inn, 4.I.1994, 1ð, au fauchage; idem, 5.I.1994, $1 \delta^{\star}$ et 3 , ces spécimens obtenus de Lycopersicon esculentum Mill. [Solanaceae]. Basse-Terre, 4.VI.1996, $3{ }^{\circ}$ et 3 , au fauchage.

Distribution: Argentine, Bahamas, Barbade, Brésil, Chili, Colombie, Costa Rica, Guadeloupe, Jamaique, Martinique, Mexique, Nicaragua, Pérou, Porto Rico, République Dominicaine, Trinidad, Venezuela, Canada et USA (Floride,...); cosmopolite.

Plantes-hôtes: espèce polyphage signalée sur 32 familles botaniques et plus de 130 genres. Les Cucurbitaceae, Leguminoseae, Malvaceae et Solanaceae sont préférées. M.

22 - Liriomyza schmidti (Aldrich)

Agromyza schmidti Aldrich, 1929:89.

Matériel examiné: Basse-Terre, 4.VI.1996, 4 đ̛ obtenus de Tabebuia pallida (Lindl.) [Bignonaceae].

Distribution: Argentine, Bahamas, Brésil, Costa Rica, Guadeloupe, Jamaïque, USA (Floride). 
Plantes-hôtes: Tabebuia pallida (Lindl.). SPENCER (1990) signale cette espèce sur 18 familles et 24 genres botaniques. A cette liste il convient d'ajouter maintenant une famille nouvelle, celle des *Thymeleaceae avec le genre *Daphnopsis, et le genre*Rauvolfia [Apocynaceae]. M.

23 - Liriomyza synedrellae Martinez

Liriomyza synedrellae Martinez, 1992: 282 (in Spencer et al., 1992).

Matériel examiné : Lynshes Gauht, 31-XII.1993, 1 đo obtenu de la plante-hôte; Fairview Inn, 31.XII. 1993, 1, 3 et 4.I.1994, 7 $\delta^{\dagger}$, tous au fauchage. Basse-Terre, 4.VI.1996, $2 \delta^{\dagger}$ et 19 obtenus de la plante-hôte.

Distribution: Guadeloupe, Saint-Martin.

Plante-hôte: Synedrella nodiflora L. [Asteraceae], M.

\section{4 - Liriomyza trifolii (Burgess)}

Oscnis trifolii Burgess : 201 (in Comstock, 1880).

Matériel examiné : Fairview Inn, 1.I.1994, 1 đ, au fauchage; Basse-Terre, 4.VI.1996, 20 et 2 우 obtenu de la plante-hôte locale.

Distribution: Argentine, Bahamas, Barbades, Brésil, Chili (île Juan Fernandez), Colombie, Costa Rica, Cuba, Guadeloupe, Guyana, Guyane, îles Vierges, Mexique, Pérou, République Dominicaine, Saint-Martin, Trinidad, Venezuela, Canada, USA (Floride,...); régions afrotropicale, australienne/pacifique, orientale et paléarctique (par introductions accidentelles).

Plantes-hôtes: *Cleome viscosa L. [Capparidaceae] à Saint-Christopher, mais l'espèce est très polyphage puisque signalée sur 45 familles et près de 160 genres botaniques, $\mathbf{M}$.

\section{Genre Melanagromyza Hendel, 1920}

\section{5 - Melanagromyza compositoides Spencer}

Melanagromyza compositoïdes Spencer, 1963: 310.

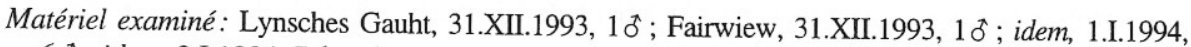

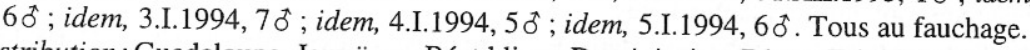

Distribution: Guadeloupe, Jamaique, République Dominicaine, Pérou, Trinidad; Venezuela.

Plantes-hôtes: *Ageratum conyzoides L., *Eclipta alba (L.) *Melanthera nivea (L.) et Rolandra fruticosa $(\mathrm{L}$.). Ces plantes-hôtes découvertes récemment appartiennent toutes à la famille des Asteraceae. La larve est mineuse de tige.

26 - Melanagromyza floridensis Spencer

Melanagromyza floridensis Spencer, 1963:312.

Matériel examiné : Fairview Inn, 31.XII.1993, 1 đ, au fauchage.

Distribution: Bahamas, Guadeloupe, Guyana, Dominique, République Dominicaine, Saint-Martin, Venezuela, USA (Floride).

Plante-hôte: les larves se développent dans les gousses de *Desmodium canum (Gmel.) [Fabaceae].

27 - Melanagromyza floris Spencer

Melanagromyza floris Spencer, 1963:313.

Matériel examiné : Fairview Inn, 31.XII.1993, $60^{\star}$; idem, 1.I.1994, $10^{\star}$. Tous au fauchage.

Distribution: Argentine, Bahamas, Chili, Costa Rica, Cuba, Guadeloupe, Jamaïque, Mexique, Porto Rico, Saint-Martin, Trinidad, Venezuela, USA (Floride).

Plantes-hôtes: Bidens pilosa L. et Calendula sp. [Asteraceae], la larve se développe dans les fleurs.

28 - Melanagromyza minima (Malloch)

Agromyza minima Malloch, 1913:328.

Matériel examiné : Fairview Inn, 31.XII.1993, 1 đ ; idem, 4.I.1994, 2 \%, au fauchage.

Distribution: Antigua, Barbade, Colombie, Costa Rica, Guadeloupe, Guatemala, Guyana, îles Vierges, Jamaïque, Dominique, Mexique, Panama, Pérou, Porto Rico, République Dominicaine, Saint-Martin, Trinidad, USA (Floride).

Plantes-hôtes : plusieurs genres d'Asteraceae, dont les Wedelia. La larve vit aux dépens des graines. 


\section{9 - Melanagromyza regalis Spencer}

Melanagromyza regalis Spencer: 164 (in SPENCER \& STEGMAIER, 1973).

Matériel examiné : Fairview Inn, 3.I.1994, 10, au fauchage.

Distribution: Chili, Guadeloupe, Dominique.

Plante-hôte: non connue.

\section{0 - Melanagromyza sp. 1}

Matériel examiné : Fairview Inn, 3.I.1994, 1 ơ, au fauchage.

Note : cette petite espèce à basiphallus en forme de $\mathrm{V}$ est identique au Melanagromyza sp. 1 signalée de République Dominicaine (in MARTINEZ et al., 1993:170) et auquel il a été comparé.

\section{1 - Melanagromyza sp. 2}

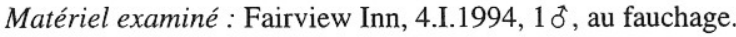

Note : petite espèce à cuillerons et franges blanches avec l'édéage à basiphallus en $\mathrm{V}$, proche, mais cependant distinct, de celui de Melanagromyza consueta Spencer.

\section{2 - Melanagromyza sp. 3}

Matériel examiné : Fairview Inn, 5.I.1994, 10 , au fauchage.

Note : cet unique spécimen est de taille moyenne. Les cuillerons sont gris à bordure et frange noires. L'édéage très sclérifié est caractéristique et ne correspond à celui d'aucune espèce connue.

Genre Nemorimyza Hendel, 1931

\section{3 - Nemorimyza maculosa (Malloch)}

Agromyza maculosa Malloch, 1913:302.

Matériel examiné : Fairview Inn, 31.XII.1993, $20^{\star}$ et $3 \%$; idem, 3.I.1994, $6 \delta^{\dagger}$ et $2 \%$. Tous ces spécimens obtenus de plantes-hôtes.

Distribution: Argentine, Bahamas, Barbade, Bermudes, Bolivie, Brésil, Chili, Colombie, Costa Rica, Cuba, Guadeloupe, Guyane, Grand Cayman, Martinique, Pérou, Porto Rico, République Dominicaine, Saint-Martin, Trinidad, Uruguay, Venezuela, Canada et USA (Floride,...).

Plantes-hôtes: cette espèce d'importance agronomique mine les feuilles de nombreuses plantes appartenant à 10 tribus dans la famille des Asteraceae. A Saint-Kitts, elle a été élevée de feuilles d'Emilia fosbergii Nicholson et de Lagascea mollis Cav. SANABRIA de AREVALO (1994) l'a obtenue de mines de feuilles de Solanum pseudocapsicum L. (Solanaceae), première mention de $N$. maculosa sur une autre famille que celle des Asteraceae.

\section{Genre Ophiomyia Braschnikov, 1897}

34 - Ophiomyia camarae Spencer

Ophiomyia camarae Spencer, 1963:326.

Matériel examiné : Fairview Inn, 31.XII.1993, 19 obtenue de Lantana camara L. [Verbenaceae]; idem, 31.I.1994, 10ิ, au fauchage.

Distribution: Argentine, Barbade, Guadeloupe, Saint-Martin, Trinidad, Venezuela, USA (Floride).

Plantes-hôtes: Lantana camara L., Stachytarpheta cayennensis Vahl [Verbenaceae], M.

35 - Ophiomyia eleutherensis (Spencer)

Melanagromyza eleutherensis Spencer: 155 (in SPENCER \& STEGMAIER, 1973).

Matériel examiné : Stone Fort River, Bloody Point, 31.I.1994, 1 đ̋, au fauchage.

Distribution: Bahamas, Costa Rica, Saint-Martin.

Plante-hôte: non connue.

\section{6 - Ophiomyia gentilis Spencer}

"Ophiomyzia" ! gentilis Spencer : 57 (in SPENCER \& STEGMAIER, 1973).

Matériel examiné: Fairview Inn, 1, 3 et 4.I.1994, 30 , au fauchage. 
Distribution: Costa Rica, Equateur, Guadeloupe, la Dominique, Pérou, République Dominicaine, Venezuela, USA (Floride).

Plante-hôte: *Stachytarpheta jamaicensis (L.) [Verbenaceae], la larve vit dans l'inflorescence.

\section{7 - Ophiomyia sp. 1}

Matériel examiné: Fairview Inn, 1, 3 et 4.I.1994, 40, au fauchage.

Note: en raison de l'absence du pinceau de soie à l'angle vibrissal, cet Ophiomyia fait partie d'un groupe d'espèces atypiques du genre. Il ne présente pas de protubérance bulbeuse entre les antennes et ressemble, à première vue, à un Melanagromyza. Cependant, la conformation de son édéage le place, dans l'état actuel de nos connaissances, dans le genre Ophiomyia. Seules des indications biologiques et notamment l'obtention de larves et de pupes permettront de mieux définir sa position générique.

\section{Genre Phytoliriomyza Hendel, 1931}

\section{8 - Phytoliriomyza jurgensi Spencer}

Phytoliriomyza jurgensi Spencer, 1983:63.

Matériel examiné : Fairview Inn, 3.I.1994, 1 ô, au fauchage.

Distribution: Costa Rica, Guadeloupe.

Plante-hôte: non connue.

\section{AUTEURS CITÉS}

Etienne J. \& MARtinez M., 1996. - Les Agromyzidae de l'île de Saint-Martin, Antilles (Diptera). Revue française d'Entomologie (N.S), 18 (4): 121-128.

- 2002. - Les Agromyzidae (Diptera) de Guadeloupe: nouvelles espèces et notes additionnelles. Nouvelle Revue d'Entomologie (sous presse).

ETIENNE J., MARTINEZ M. \& HUC A., 1993. - Les mouches mineuses, (Diptères Agromyzides) d'importance économique aux Antilles. ANPP, Troisième Conférence Internationale sur les ravageurs en Agriculture. Montpellier 7-8-9 décembre 1993, II: 1261-1272.

Garces Gonzalez G., 1988. - Presencia en Cuba de Calycomyza bahamarum Spencer (Diptera: Agromyzidae) con notas sobre su biologia. Miscelanea Zoologica, Havana, $n^{\circ} 38: 2-3$.

MARTINEZ M., 1994. - Japanagromyza etiennei n. sp (Diptera, Agromyzidae) ravageur potentiel des Phaseolus spp. (Leguminosae) dans les Caraibes. Revue française d'Entomologie (N.S), 16 (2): 81-85

MARTINEZ M. \& ETIENNE J., 1991. - Les recherches faunistiques en Guadeloupe. Bilan général et cas des Diptères Agromyzidae. Rencontres Carä̈bes en Lutte Biologique, Guadeloupe, 5-7 novembre, 1990: 77-84. Ed. INRA, 1991 (Les Colloques, n58).

- 2002. - Liste systématique et biogéographique des Agromyzidae (Diptera) de la région néotropicale. Bollettino di Zoologia agraria e di Bachicoltura, Ser. II, 34 (1):25-52.

MARTinez M., EtiEnNE J., AbUd-ANTUn A. \& ReYeS M., 1993. - Les Agromyzidae (Diptera) de la République Dominicaine. Bulletin de la Société entomologique de France, 98 (2): 165-179.

SANABRIA DE AREVAlo I., 1994. - Insectos minadores (Diptera, Agromyzidae) de la Sabana de Bogota (Cundinamarca, Colombia). Revista Colombiana de Entomologia, 20 (2):61-100.

SPENCER K.A., 1973a. - Les Agromyzidae (Diptera) of economic importance. Series Entomologica, 9. The Hague: W. Junk, 418 p.

1973b. - The Agromyzidae (Diptera) of Venezuela. Revista de la Facultad de Agronomia, Universidad Central de Venezuela, Maracay, 7 (2):5-108.

1990. - Host Specialization in the world Agromyzidae (Diptera). Series Entomologica, 45, Kluwer Academic Publishers, Dordrecht, Netherlands, XI, 444 p.

SPENCER K. A. \& STEGMAIER C.E., 1973. - The Agromyzidae of Florida, with a supplement on species from the Caribbean. Arthropods of Florida and neighboring land areas. Florida Department of Agriculture and consumer services, Gainesville, 7: 1-205.

SPEnCER K. A., MArtinez M. \& Etienne J., 1992. - Les Agromyzidae (Diptera) de Guadeloupe. Annales de la Société entomologique de France (N.S.), 28 (3):251-302. 
Analyse d'ouvrage, Fabre: le miroir des Insectes de Patrick Tort, par Yves Cambefort (suite de la page 80)

tique de concepts différents mais apparentés». Outre son style, le comique (involontaire?) de ce passage tient au fait qu'il dénonce ce que Patrick Tort fait lui-même tout au long de cet ouvrage! Ne pouvant relever ici tous les passages prêtant à critique, voire à discussion, je me contenterai d'en signaler deux. D'abord, on peut prendre l'auteur en défaut sur son terrain de prédilection: l'œuvre de Darwin. Patrick Tort examine la fameuse appréciation de celui-ci: «M. Fabre, cet observateur inimitable». Constatant qu'elle est souvent citée mal à propos, que son origine exacte est méconnue, bref que l'ignorance bien française concernant Darwin est à l'œuvre une fois de plus, il décide de rectifier ces erreurs. Aux p. 148-152, il examine la chronologie de la sentence dans les éditions anglaises et allemandes de l'Origine des espèces, mais il ne cite pas les éditions françaises. Or, du point de vue de Fabre (qui cite plusieurs fois l'appréciation de Darwin), ainsi que du lecteur français de Tort, il était plus intéressant de connaître son origine dans notre langue, ce dont il ne nous est rien dit. En fait, l'appréciation darwinienne apparaît en français dans la $2^{\mathrm{e}}$ édition de la traduction de l'Origine par Clémence Royer (novembre 1865, p. 104), environ un an avant son apparition dans la $4^{\mathrm{e}}$ édition anglaise (fin 1866). On dira que tout ceci n'a guère d'importance, et j'en serai d'accord; mais Patrick Tort le "monte en épingle" d'une façon qui tombe à plat, assez drôlement, par suite de sa méconnaissance des versions françaises de Darwin. Celles-ci ont pourtant une grande importance du point de vue de l'histoire des sciences, puisque ce sont elles qui ont permis au public français du $\mathrm{XIX}^{\mathrm{e}}$ siècle de connaître les travaux du savant anglais, et, suivant les cas, de les accepter ou, plus souvent, de les rejeter. Curieusement, Tort accuse Fabre à plusieurs reprises d'avoir une documentation insuffisante, de ne pas lire les auteurs, etc. Il n'invente rien: Fabre lui-même déclare qu'il préfère ignorer ce qu'écrivent les autres afin de ne pas fausser son jugement. En réalité, les Souvenirs entomologiques citent environ 80 auteurs, anciens et modernes, et notamment les principaux entomologistes des XVIII et XIX ${ }^{\mathrm{e}}$ siècles: on voit que cet "aveu" de Fabre n'est pas toujours supporté par les faits. D'ailleurs, Patrick Tort se rend coupable lui aussi, à plusieurs reprises, de ce péché d'ignorance. Outre les traductions anciennes de Darwin, il ignore certains travaux importants consacrés à Fabre. Par exemple, les remarquables publications d'Ido Yavetz $(1988,1991)$, ainsi que les ouvrages plus anciens de Bujeau (1943), Revel (1951), ou encore Lamore (1969), sont absents de la bibliographie et de l'index de l'ouvrage.

Une autre vétille me semble assez démonstrative de la façon dont fonctionne l'ouvrage (c'est-à-dire partir de prémisses erronées, puis en tirer des conséquences ou des conclusions qui le seront nécessairement aussi). A la p. 295, il s'en prend, on ne sait pourquoi, à la devise de Fabre: De fimo ad excelsa, qui ne lui agrée pas «et dont la version correcte aurait été sans doute $\mathrm{Ab}$ infimo ad excelsa». Pour Tort, cette devise «marque les limites de l'autodidactisme de Fabre en matière de langues anciennes» et manque singulièrement "de grâces littéraires et d'égards envers ses ascendants»! En fait, la formule est parfaitement conforme à la langue latine la plus classique: le mot fimus est employé par les meilleurs auteurs, en particulier Virgile, que Fabre connaissait bien. Le sens de sa devise («Du fumier à ce qu'il y a de plus élevé») résume exactement la démarche fabrienne: il est possible de trouver dans les lieux les plus humbles, voire les plus vils, les vérités les plus hautes. On peut y voir aussi une allusion au scarabée, insecte coprophage et néanmoins "sacré", que Fabre a fréquenté toute sa vie. D'ailleurs, le mot fimus est employé par Linné à propos des scarabées, au début de la partie "Insectes" du Systema Naturae: «Scarabaorum Larva vivunt tranquilla sub terra; harum pleraque fimo delectantur \& eo pascuntur» [Les larves de scarabées vivent paisiblement sous la terre; la plupart d'entre elles se plaisent danș le fumier et s'en nourrissent]. Fabre connaissait bien le Systema Naturae, qu'il cite à plusieurs reprises. Les scarabées figurent à la première page des premiers Souvenirs entomologiques (1879), peut-être en hommage à Linné. Ce sont d'ailleurs les Coléoptères qui occupent le premier rang (en nombre de pages) dans l'ensemble des dix séries des Souvenirs entomologiques. 